\title{
Prediction of Future State Based on Up-To-Date Information of Green Development Using Algorithm of Deep Neural Network
}

\author{
Liyan Sun (D), Li Yang, and Junqi Zhu \\ School of Economics and Management, Anhui University of Science and Technology, Huainan 232001, Anhui, China \\ Correspondence should be addressed to Liyan Sun; 2011066@aust.edu.cn
}

Received 4 March 2021; Revised 5 April 2021; Accepted 19 April 2021; Published 8 July 2021

Academic Editor: M. Irfan Uddin

Copyright ( 92021 Liyan Sun et al. This is an open access article distributed under the Creative Commons Attribution License, which permits unrestricted use, distribution, and reproduction in any medium, provided the original work is properly cited.

\begin{abstract}
In this study, the focus was on the development of green energy and future prediction for the consumption of current energy sources and green energy development using an improved deep learning (DL) algorithm. In addition to the analysis of the current energy consumption used for the natural gas and oil as fuel, deep neural network algorithm is used to train the system as well as to process the data obtained previously, ranging from literature from the year 2003 until the year 2019, for consumption of fuel. Also, using the proposed algorithm to predict the development of green energy consumption till 2030 is presented in terms of solar and wind generators. The resulting study also focuses on depletion of energy currently used or pollution caused because of it. The green energy controlling issue can take effect by using multiple layers of handling different features extracted from different sources and then learning the system to control it.This study aims to take advantage of carbon emissions to reduce their impact and dependence in the future on environmentally friendly renewable energies. Predicting the correct and precise amount of energy consumption and increasing the amount of environmentally friendly energy lead to a healthy ecosystem. The expected green energy consumption in the future is almost $78.25 \mathrm{EJ}$ in 2030 and will be, in total energy average, $56 \%$ in 2045 . The aim is to reduce dependency on costly and environmentally harmful fuels.
\end{abstract}

\section{Introduction}

Currently approved energy sources are petroleum, coal, and natural gas, while the alternative green energies that will be relied upon in the future are solar and wind energy which may be important in different life fields such as medical, industrial, military, and social applications [1]. However, increasing consumption of green energy always contributes to sustainable development. Many countries have set goals for Renewable Energy (RE) or green energy [2] as well as to reduce pollutants suffered by the ecosystem. Many of the determinants were previously preventing some countries from using alternative energy, but with technological development and modern technologies, they have become available to many countries [3]. The low cost of green energy has encouraged many countries to use it as an alternative or at least an assistant to reduce energy consumption dependent on carbon fuels. Globally, the increase in the percentage of the population is reflected in the increase in the demand for energy sources, which in turn leads to an increase in global energy consumption, which leads to environmental degradation day after day [4]. Influence resulting from increasing the energy consumption leads to a negative impact on human health and the environment. Among the reasons stated above, it is necessary to limit the consumption of carbon sources and to encourage the production of green energy and clean technology [5]. Alternative energy is an unavoidable choice, and in return, there will be proper management for the safety of consumption and carbon sources. Green energy has many sources such as wind, solar, tidal, biomass, and hydroelectricity, as shown in Figure 1.

Prediction of efficiency energy increased in subjected manner according to international attention [6]. Many researchers have studied regarding energy development of efficiency and energy-saving techniques in different perspectives. Utilization efficiency is important for reducing energy consumption and decreasing the demand on the power for both the present day and future. Energy in general 


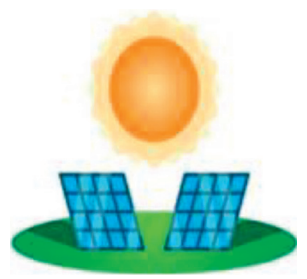

(a)

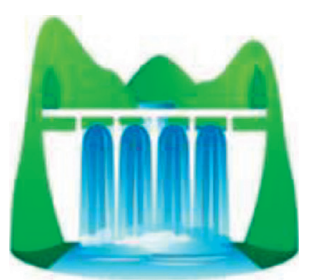

(b)

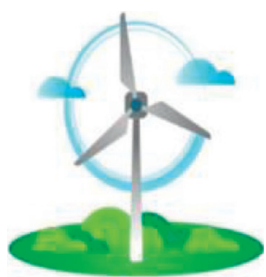

(c)

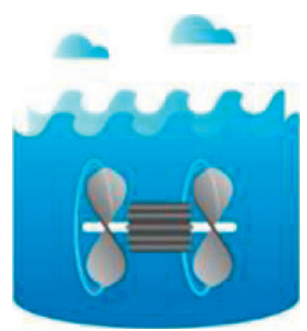

(d)

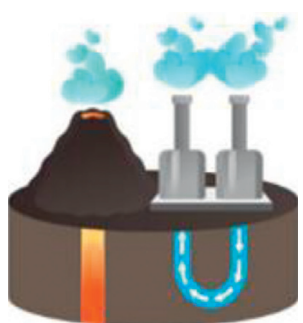

(e)

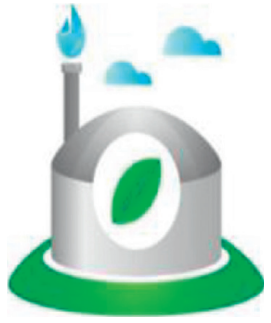

(f)

Figure 1: Types of green energy. (a) Solar. (b) Hydro. (c) Wind. (d) Tidal. (e) Geothermal. (f) Biomass.

characterizes as high consumption, high pollution, and low efficiency. The two main aspects needed for improving energy are as follows: firstly, determining the critical factors that control energy efficiency and, secondly, quantifying the degree of influence. These factors majorly affect the consumption fuel cost in the world, especially in nonpetroleum countries. Most of the time, developing energy is thought of when traditional energy sources become more productive and when the economy grows in terms of industries, borders, and environmental policies. [7]. Modern countries with a big part of industries use alternative energy, namely, in the USA, solar energy is considered the main source of power consumption in California, and $24 \%$ of power resources in Germany use wind turbine generators to supply the people [8]. Even the petroleum countries such as Saudi Arabia and Qatar try to use green energy in different fields and avoid exhausting oil energy. In 2016, 167 countries made an agreement to use renewable energy, or the so-called green energy. These efforts became fruitful, especially when China joined the agreement. On the other hand, there should be commitments in terms of energy consumption and the pollutants that emanate from it. Some of these efforts were in vain due to the increase in global manufacturing rates, especially to meet the needs of the global market. Global attention focused largely on the impact of an environmental alternative energy source fossil fuel [9]. A different point of view considers the impact between many sources of renewable energy; however, a renewable energy source has low emission of carbon. Many sources of renewable energy such as wind turbines, bioenergy, and solar Photovoltaic (PV) can be used with high degree of efficiency.

Cost of renewable energy varies depending on the source; every year, more studies arise regarding this issue to help reducing the cost [10]. In general, the price difference and its variance remain constant in terms of the limits, while the decrease in the cost of one type is accompanied by a decrease in the other types at the same level, as shown in Figure 2.

Recently, new parametric methods have been applied to predict the efficiency of energy. Data-driven methods such as Principle Component Analysis (PCA) and statistical frontier techniques are used for establishing evaluation of energy efficiency [11]. In many interesting studies of improving energy efficiency, machine learning is used to extract useful information from big data when modeling the system managements. Data mining methods such as neural network and regression analysis are used for optimizing energy efficiency [12]. In this study, we try to collect data of vary factors which are not comparable units. Data from the literature were used as a dataset for training each suggested machine learning method. Many studies related to China's energy efficiency used data mining techniques to predict future efficiency of the energy [13]. For normal machine learning and data mining algorithm, a comparing method is not used for feature extracted due to its simple nature, so the final prediction was not accurate. For this reason, feature extraction is considered in this study to manipulate them before processing, as a preprocessing stage. For good processing, we have to select features carefully and comprehensively consider the selected factors before processing in deep learning algorithm. The reason of considering renewable energy is that it is the most promising alternative for traditional fuel (fossil) as it is naturally replenished in different geographical places, clean, and green. As it is a renewable energy, it is unlimited in place and time and can be used in any region and does not require the cost of transportation from one place to another. One of the important reasons is that alternative energy is easy to convert to electric energy and the conversion cost is low compared to the rest of the sources. The development in this field proves that the alternative energy is subject to dramatic increase, and in the future, it may be sufficient to meet the need for it. No matter 


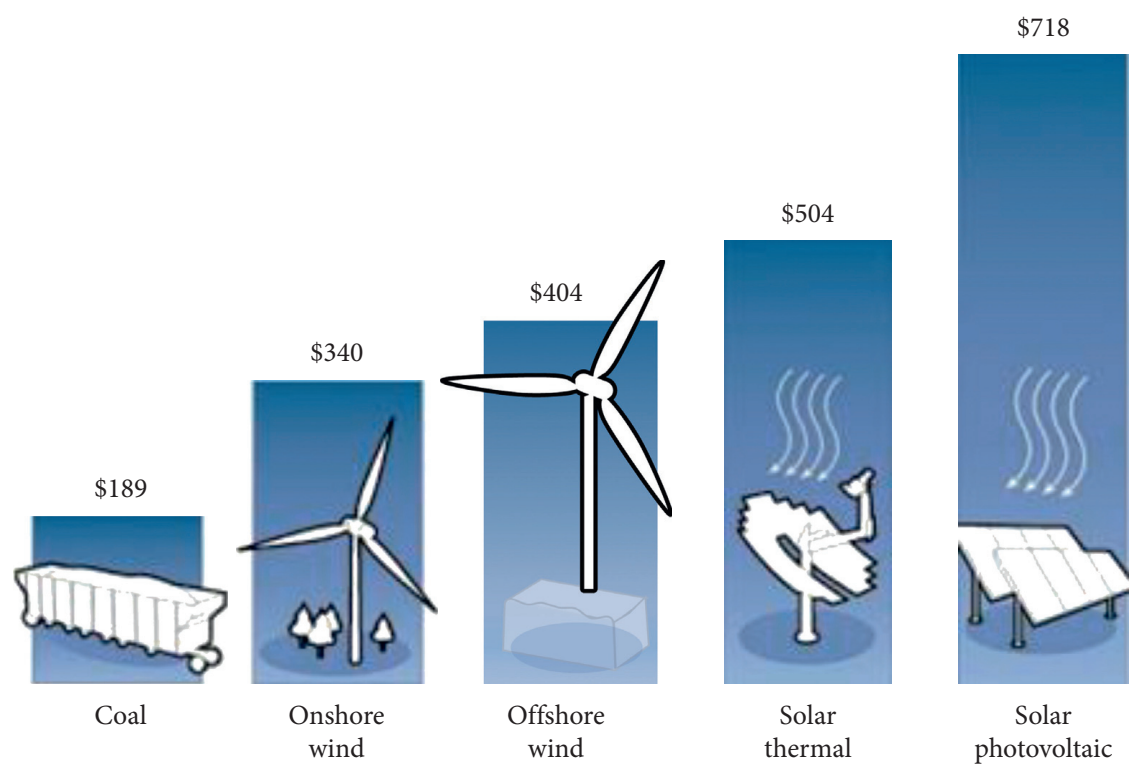

FIGURE 2: Variance of the price in alternative energy.

how much the energy costs, whether alternative or conventional, the main element is management and control. From this, we notice previous studies attaching the utmost importance to the management side, which leads to an increase in the efficiency of any system. Prediction for energy efficiency may provide accurate results for few hours or even few days, but for long time, the system needs to deal with artificial intelligence and use one of the deep learning algorithm to estimate parameters that effect for future prediction [14]. Right prediction is not easy; the best long right prediction was achieved in [15], as shown in Figure 3.

According to the reported studies, renewable energy such as the three categories in Figure 3 (solar thermal, smallscale PV, and solar PV) spreads widely, according to expectations and prediction [16].

Accordingly, deep learning algorithm has attracted more attention nowadays. This is because of the three main features, strong generalization, unsupervised learning of extracted features, and training of a big dataset collected from the literature. Compared with shallow techniques that do not use deep learning, the alternative model is naturally widely used and implemented in image processing, pattern recognition, classification, and fault detection. One of the deep learning approaches used in wind generation used collection of data to make the system more stable and predictable. For obtaining forecasting in energy, extracting the informative data is very necessary, and automatic extraction of the features affects directly the system results. In [17], an integrating method that depends on deep belief network with grey theory is proposed to estimate preday ahead PV output power and superior efficiency achieved during benchmarking. A new deep learning method was developed by Li et al. [18] to predict wave energy short time, and an encouraging result was achieved which was very helpful in real time but inaccurate with long time. Many deep learning approaches are used in the literature; each has its own contribution such as evaluation in deep neural

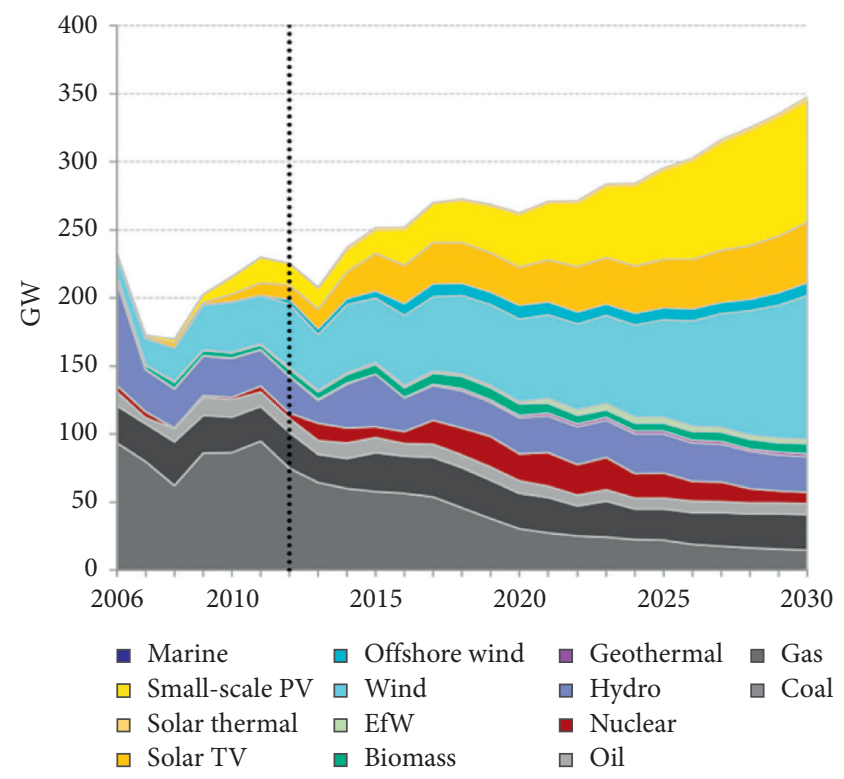

FIgURE 3: Prediction for most important energies used in the world (predicted successfully till 2020).

network [19], recursive od deep neural network [20], and learning machine based on stacked extreme [21], reporting for forecasting of renewable energy. Good stability, accuracy, and effectiveness reported in [22] by using deep learning depend on forecasting. The energy system used was beneficial in scheduling, planning, and management [23].

In recent day, green development energy remains active in the literature by several studies which have been published. Power generation (PV) was reviewed in [24] using the forecasting technique in terms of the time series and physical method, while Rahim et al. [25] made an extensive overview of multiple techniques in PV green energy in short term. A big survey was presented in [26] on generating wind energy 
with their benefits and the most expected costs for the system. A systematic review [27] introduced two categories, solar and wind energy generation, and explained the state of the art on energy prediction.

This manuscript is structured as follows: Section 1 is introduction, Section 2 provides a detailed literature review, and Section 3 gives the reason of using these resources. Section 4 explains the deep learning strategy followed by Section 5 which shows the relation between these two issues. Section 6 shows the features extracted in the system followed by Section 7 which details the method used. All results gathered by the system are explained in Section 8. Finally, Section 9 gives the conclusion of this research.

\section{Literature Review}

Wind energy generation has become an important topic, especially in islands and coastal areas, and managing and controlling such a network requires systems that are similar to artificial intelligence, such as deep learning [28]. Using real-time variables directly with the wind energy generation system increases the efficiency of the system and, thus, increases the energy production; the backpropagation technique [29] and the deep-learning-based ensemble approach used for renewable energy such as wind energy generation achieved good result in terms of accuracy for online monitoring [30]. A hybrid model that combines machine learning with a statistical method is used to solve the Solar System of renewable energy [31] of using deep learning to control the parameters of the Solar System. A mixture density network (MDN) was used in [32] to control photovoltaic (PV) systems and extract important features that help to predict the energy in the later time.

\section{Why Green Development}

Continuous support for the United Nations and the authorities in charge of climate change, which has begun to deteriorate, as well as financial assistance, has surpassed one trillion dollars, to stop environmental pollution that has harmed human existence.

Global investment in clean energies has reached its peak due to the neglect of some countries, especially the thirdworld countries and countries with a high population density such as India, to the ongoing depletion of environmental resources and pollution, which led to an increase in the temperature of the atmosphere.

A few years ago, the International Energy Agency confirmed that the cost is close to a trillion dollars every year in creating new infrastructure in 2030 to change the economy from an economy that depends entirely on oil to generating clean energy and environmentally friendly technologies that help keep the temperature from rising above the dangerous limit $3.6 \mathrm{C}^{\circ}$.

Many prominent personalities and global investors confirmed to increase their investments in clean energy during a meeting at the United Nations organized by the
Ceres Investment Network. In the previous year, \$ 300 billion was spent, but at the same time, twice that amount was invested in fossil fuel exploration. Therefore, we conclude that the ratio is not reasonable, and on the contrary, the ratio must be the opposite.

\section{Deep Learning}

The development in data science and modern technology such as big data and high-performance computers has provided an opportunity for machine learning to understand data and their behavior through complex systems. Machine learning gives the machine the ability to learn in different algorithms without strict orders from a certain program or limited instruction [33].

Deep learning can be defining as a technique of machine learning to learn useful features directly from given different media or problems. Many layers were exploited by deep learning for nonlinear data processing of unsupervised or supervised feature extraction for classification and pattern recognition [34]. Deep learning motivation is greatly reduced by the Artificial Intelligence (AI) area, which simulates the ability of the human brain in terms of analyzing, making decision, and learning. Deep learning's goal is to emulate an approach of hierarchical learning of extracting features by the human brain directly from unsupervised data.

The core of deep learning is hierarchially computing the features and representation information, such as defining the features starting from the low level to the high level. With huge data which come from energy sources, the standard techniques of machine learning do not work well when running directly due to the ignoring nature of data behavior. In deep learning, features are extracted automatically from the given energy system. The characteristics of this method of features considered one of the learning methods in the system [35].

Characterization of such input data used as a feature is the key issue to the success of processing prediction of the future state. There are limitations for extracted features in the green energy system such as scheduling time and the amount of consumption ratio based on total demand [36]. For this reason, we can use deep learning by its feature extraction to solve the limitations in such systems.

As mentioned before, the main difference between machine learning and deep learning is the feature selection method, as shown in Figure 4.

Features in deep learning are generated automatically to simulate the appropriate results [37]. Different hidden layers participate in making the decision by using the feedback from the next layer to the previous one or the resulting layer will have to be fed into the first layer [38]. DL enables computers to be able to perform complex calculations by relying on simpler calculations to optimize computer efficiency. It is difficult for a computer to understand complex data such as collection data from the literature or a series of data of a complex nature, so we use deep learning algorithms instead of usual learning methods [39]. 
A simple neural network
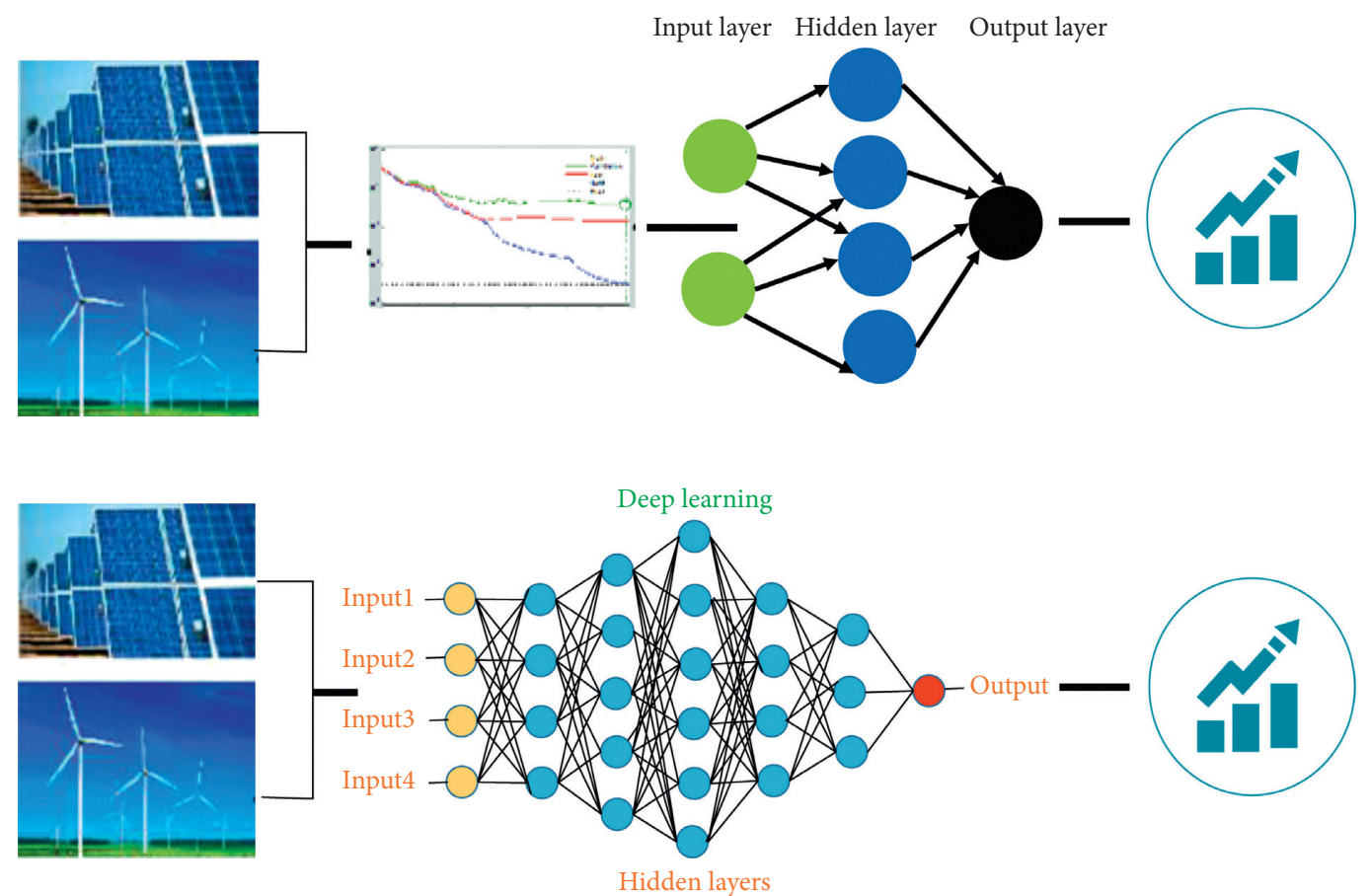

Figure 4: Difference of simple neural network and deep learning in feature combination techniques.

\section{Structure of Deep Learning in Green Development}

We present in this section the basic structure considered in deep learning, which is important to improve the accuracy for renewable energy prediction. In general, there are three main deep learning types, namely, deep belief network, auto encoder, and recurrent deep neural network. Also, learning machine of stacked extreme, deep learning reinforcement, and convolutional deep neural network that depends on the prediction model are reported.

5.1. Stacked Auto Encoder. It can be defined as a neural network feedforwarding including multiple hidden layers to an auto encoder. In a way, the output of each layer must be connected to the input of the successive layer, and the auto encoder normally consists of an encoder and decoder. Figure 5 illustrates the structure.

When considering unsupervised machine learning, the input will take $u \in R^{d}$ while hidden layers produce $y \in R^{d}$ as a latent map, so the decoder $u$ will be the same as the encoder $u$ in terms of weight and both are stored in vectors, and during training, it minimizes the error reconstruction according to the given present assumption of the input space. Square error and entropy are used as the method for minimizing function. The encoder and decoder behave as nonlinear transformation, and this is actually considered as the feature extracted within a system.

5.2. Deep BeliefNetwork. Deep belief network was developed first by Geoffrey et al. [40], which is applied in many areas. It

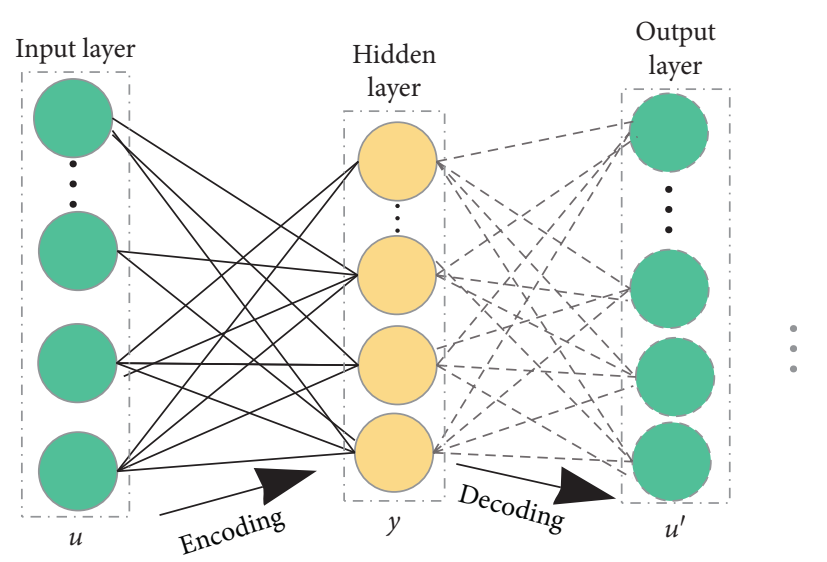

Figure 5: Structure of stacked of the auto encoder.

generates a model of the graphical issue, simple composition, and unsupervised data, for example, for Boltzmann restricted machine of symmetrical and bidirectional connection in neural network within its layers [41]. It consists of a Boolean neuron as visible and many hidden layers, where hidden and visible layers are denoted as $h$ and $v$ with $b$ and $a$ which are the respected biases, as shown in Figure 6.

5.3. Recurrent Deep Neural Network. The recurrent deep neural network is derived from the recurrent neural network. That is one of the classes of artificial neural network that satisfy the connection of given nodes and can create a direct graph [42]. It is a strategy that affects the feedback connection on the neural system as time series called a recall 


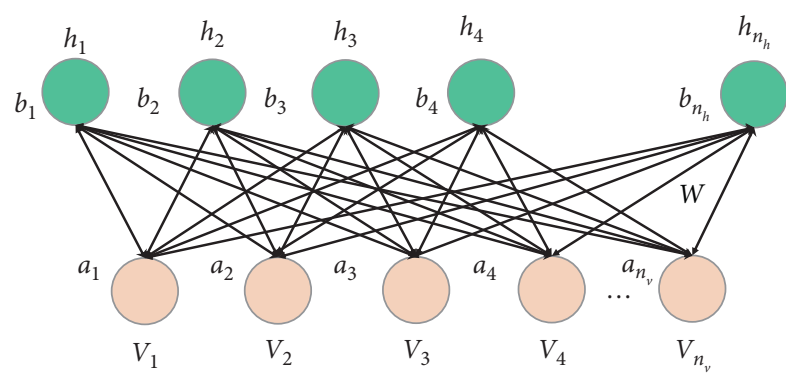

Figure 6: Boltzmann machine learning.

to change the neural state from the previous node. A typical RNN structure is given in Figure 7. Unlike the traditional feedback of a node to the whole system, it can feedback not only to the same node but also to the whole system. This is worthy with big data derived from the previous experiment and helpful in forecasting of renewable energy. Deep RNN can be formulated in four different ways: first, it can learn the nontemporal structure given from the input deepening to the hidden function. In this way, the relation between features is expressed more easily. The second way is to deepen in the side of hidden to the output function. This way allows compacting the layers more than usual. This way is useful when keep previous history and fulfilling for real-time prediction. The third way is hidden to hidden connection by adding the data source to the sum of the previous inputs, which is called the hidden state of fixed length. In this way, the hidden layer can adapt any pattern rapidly from the input design and useful summary always participates in updating the results which are replaced with the following: "it plays an important role in determining the results of the system." The fourth way is staking of multiple hidden layers recurrent; in this way, each layer can run in different modes in different times and can estimate the different times for the input and make decision for each layer.

\section{Feature Extraction for Green Energy}

The term of efficiency in energy world refers to less usage energy when producing the same amount of services or, in other words, useful output. It can be presented as follows:

$$
\text { energy efficiency }=\frac{\text { useful output of a process }}{\text { energy input of a process }} \text {. }
$$

Its range would be $[0,1]$. Efficiency is affected by different vectors. So, the critical factor is identified in advance. The efficiency energy is 0 when it is less than 0.5 ; otherwise, it is 1 , which means high energy efficiency. There are other features such as energy consumption, energy investment, labor force, and capital input that are selected in advance as input factors. While the output factor is pollution of the environment, many factors can be extracted as follows:

(i) Primary output energy (F1) is the product that exploited energy resources during collecting report. Coals, oil, and gas from the mine or fields considered primary features, as well as wind and solar.

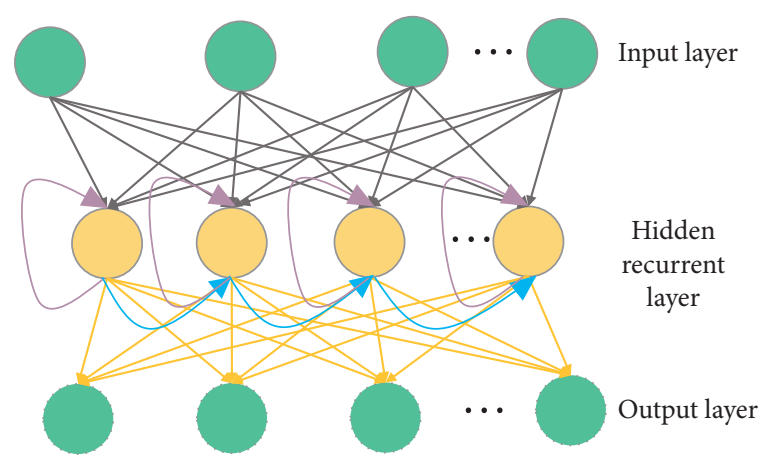

FIGURE 7: Recurrent neural network type.

(ii) Total energy consumption is (F2) is the amount of energy used as a physical quantity that is put in the report in advance, or it can be said the useful energy gain from the system.

(iii) Elasticity coefficient of consumption (F3) can be defined as the ratio of energy consumption and national growth given in the following equation:

$$
F 3=\frac{\text { average rate of energy consumption }}{\text { average rate of national growth }} \text {. }
$$

(iv) Gross local product (F4) refers to the measurement of aggregation production. That is the sum of all institution and resident units within production.

(v) Investment of the energy industry (F5) refers to the total industry investment.

Selecting features always determined by the dataset which is already collected by the previous work, $x_{i}$ can be considered as a value in the dataset with item $i$, and the data transform by transformation function is as

$$
x^{*}=\frac{x_{i}}{\sum_{k=1}^{n} x_{k}} .
$$

A positive dataset manipulates and simply results in the standard $[0,1]$, and due to the error, it can be easily eliminated by this function.

Selecting features affect directly the accuracy, scalability, and efficiency of learning. $\mathrm{F}$ is considered as the features extracted in advance, $\mathrm{C}$ is the efficiency of energy with two cases high and low, and the gain $\mathrm{G}$ can be calculated as

$$
\begin{aligned}
I G\left(F_{j}, C\right)= & H(C)-H\left(\frac{C}{F_{j}}\right)=-\sum_{i=1}^{2} p\left(C_{i}\right) \log _{2} p\left(C_{i}\right) \\
& +\sum_{j=1}^{m} \sum_{i=1}^{2} p\left(F C_{i j}\right) \log _{2} p\left(F C_{i j}\right),
\end{aligned}
$$

where $I G\left(F_{j}, C\right)$ refers to information gain of $F_{j}$ and $H(C)$ is the entropy. 


\section{Proposed Method}

For each energy development, the gain will be the efficiency and prediction class. Any system in machine learning starts with collecting data from the standard dataset, but in our case, the dataset will be information provided in the previous year which is real as estimation of the system when learning should be available in advance to evaluate the system. Data considered for both traditional energy and green energy were applied previously, so the given result will be compared with the simulating system to find the efficiency. The set of features provided to the system of deep neural network is shown in Figure 8.

Features input to the system each with their weights are collected. The weight of the selected feature specifies whether to control the priority of the feature and determine if a certain feature affets the system output or not. $F(u)$ is the function that is responsible for establishing a hidden layer according to the features.

Figure 9 shows the stages for the training system; the first is the preparation of the renewable energy supported to the system. Different data are input to the system from wind, solar, etc. The deep neural network model considered in this study is most suitable for training and testing. Multi-inputs of the nonlinear process run in terms of feature and its weight. The backpropagation network is improved in this study to adapt with energy development. Feed forward is the strategy to make the DNN effective and suitable. Many evaluation runs when training the system and procedures are as follows:

(1) Net input evaluation of the $j$ th node and the node net input of the $k$ th number of the hidden layer:

$$
\text { net }_{j}=\sum_{i=1}^{n} w_{i j} x_{i}-\theta_{j}, \text { net }_{k}=\sum_{j} w_{k j} x_{j}-\theta_{k},
$$

where $i$ is the input node, $j$ is the hidden layer, $k$ is the output layer, $w_{i j}$ is the weight connection, $w_{k j}$ is the weight to the output layer, and $\theta_{k}$ is the threshold between layers

(2) Output evaluation of $j$ th hidden layer nodes and the $k$ th output layer:

$$
h_{j}=f_{h}\left(\sum_{i=1}^{n} w_{i j} x_{i}-\theta_{j}\right), h_{j}=h_{k}\left(\sum_{j} w_{k j} x_{j}-\theta_{k}\right) \text {, }
$$

where

$$
f_{h}(x)=\frac{1}{1+e^{-\gamma h x}}, f_{k}(x)=\frac{1}{1+e^{-\gamma k x}}
$$

Here, $h_{j}$ is the hidden layer vector, and $y_{k}$ is the neuron of the output layer. $F_{h}()$ and $f_{k}()$ are the logistic activation functions of transform from the input layer to the hidden layer and then to the output layer. $\gamma k$ and $\gamma h$ control the slop function.

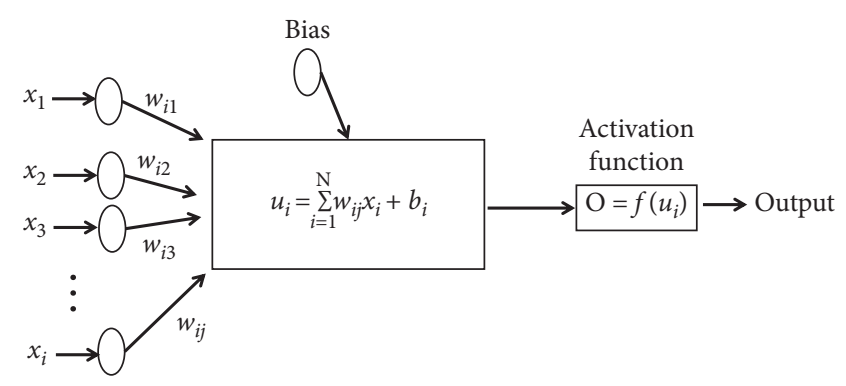

FIGURE 8: Features in the DNN of the proposed system.

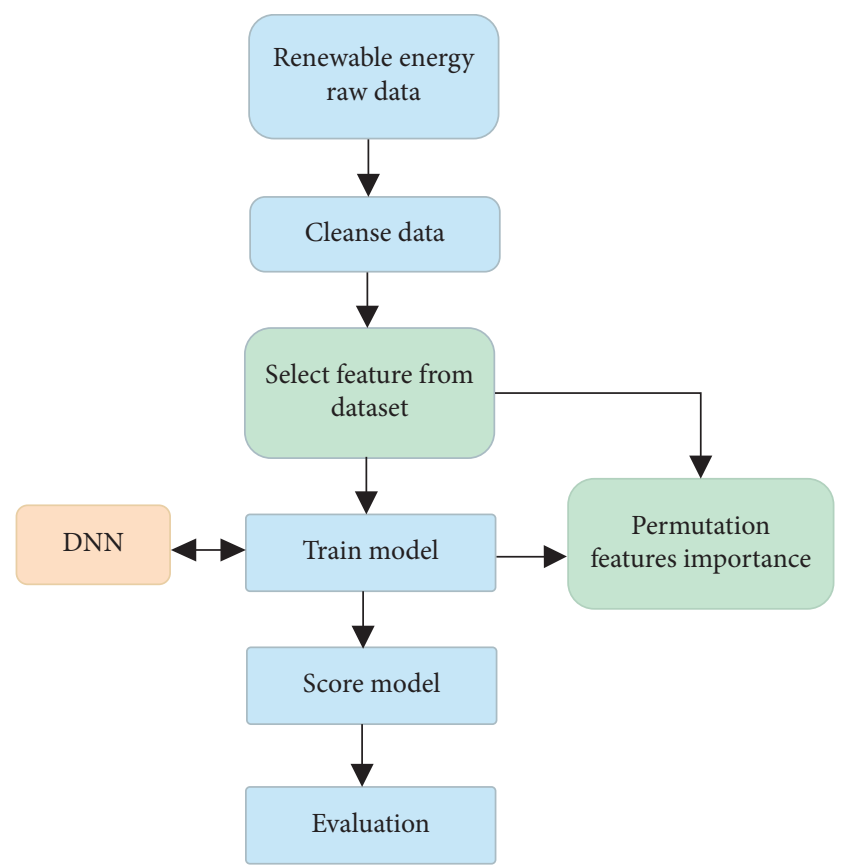

Figure 9: General flowchart of the system.

The DNN actually is the part of the management system that controls the green development. Prediction of the future state can be controlled by the DNN, but the whole system is used to increase the efficiency of the energy system, as shown in Figure 10.

The most important issue in developing green energy is how to predict the future state and efficiency of the results.

Most of the existing studies in the field of clean energy tend to use modern algorithms to control systems. The difference in most studies comes from the new proposed extracted features and how they are compatible with the algorithm used. Generating and managing clean energy is completely different from the methods of managing recycled waste to generate energy.

\section{Experimental Results}

A fabulous dataset is used in training the proposed system. This dataset consists information of the previous state with resulting estimation to mark it as a label for the training mode. 


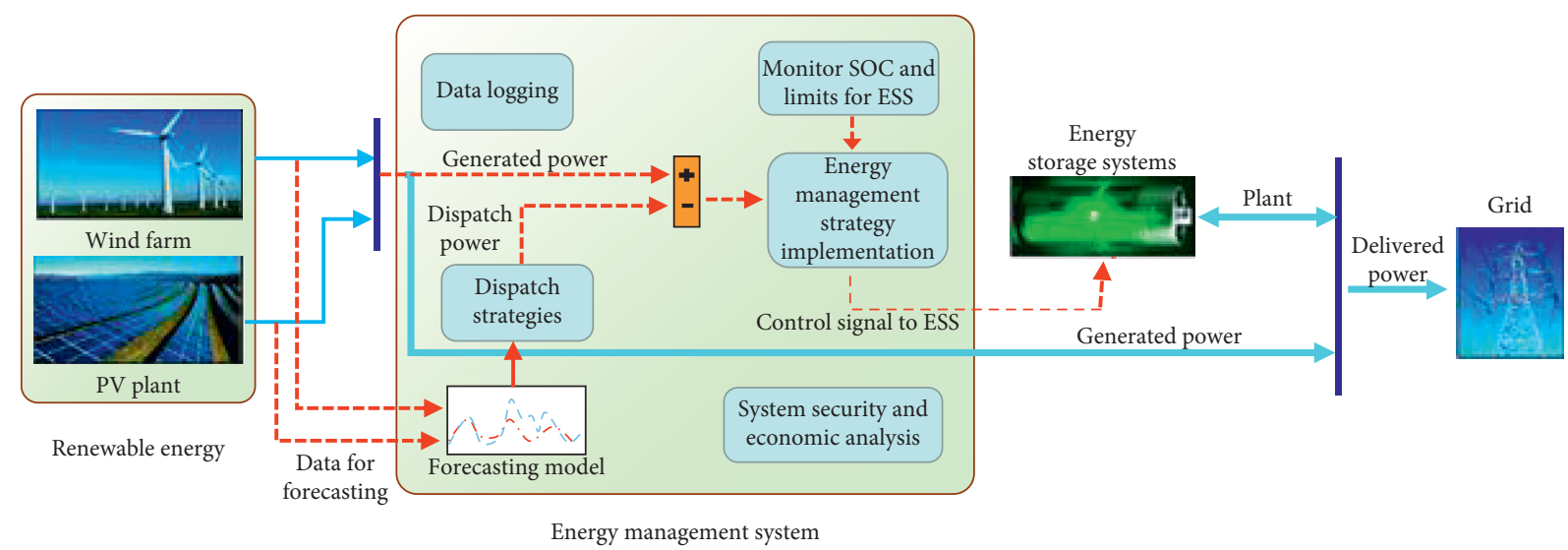

$\longrightarrow$ Power flow
$-\rightarrow$ Data/control flow

FIGURE 10: Comprehensive green energy system including management and control of two types of green energies, PV and wind tower.

TABLE 1: Result of traditional energy consumption.

\begin{tabular}{|c|c|c|c|c|c|c|c|c|}
\hline Year & 2009 & 2012 & 2016 & 2019 & 2021 & 2025 & 2028 & 2030 \\
\hline \multicolumn{9}{|c|}{ Oil cons. } \\
\hline Actual & 102.3 & 106.5 & 107.6 & 110.5 & 112.6 & 116.8 & 119.8 & 122.3 \\
\hline DNN & 102.2 & 104.3 & 106.8 & 109.4 & 111.2 & 113.6 & 116.1 & 120.9 \\
\hline AMRE & 0.99 & 6.66 & 6.54 & 2.69 & 1.23 & 7.16 & 7.21 & 4.25 \\
\hline$R^{2}$ & 0.9954 & 0.9925 & 0.9978 & 0.9991 & 0.9994 & 0.9982 & 0.999 & 1.000 \\
\hline \multicolumn{9}{|c|}{ Natural gas cons. } \\
\hline Actual & 68.3 & 70.3 & 78.6 & 79.3 & 84.21 & 88.4 & 92.4 & 93.1 \\
\hline DNN & 67.5 & 68.5 & 76.5 & 77.5 & 82.94 & 86.92 & 91.45 & 92.94 \\
\hline AMRE & 5.4 & 7.56 & 7.2 & 1.9 & 4.2 & 0.6 & 1.9 & 1.6 \\
\hline$R^{2}$ & 0.9954 & 0.9925 & 0.9978 & 0.9991 & 0.9994 & 1.00 & 0.999 & 0.994 \\
\hline \multicolumn{9}{|c|}{ Primary energy cons. } \\
\hline Actual & 209.2 & 212.2 & 220.9 & 282.6 & 278.21 & 317.5 & 345.2 & 398.7 \\
\hline DNN & 199.9 & 209.6 & 218.4 & 271.6 & 272.45 & 312.96 & 341.65 & 391.21 \\
\hline AMRE & 5.11 & 5.96 & 8.23 & 5.06 & 4.85 & 0.95 & 1.25 & 0.06 \\
\hline$R^{2}$ & 0.9954 & 0.9925 & 0.9978 & 0.9991 & 0.9994 & 0.995 & 1.00 & 0.994 \\
\hline
\end{tabular}

In this study, the DNN of green energy development consumption is presented, in addition to analyses of the traditional primary energy consumption. The number of hidden layers for the DNN is used to set the node for each one. Feed forward and back propagation are performed for the neural network. The neural network trains with two main inputs, year and actual consumption, and sets the learning rate with momentum coefficient 0.8 to process learning. For good fits, 80000 iterations are used until satisfying results were obtained. Two main validation parameters used, which are Absolute Mean Relative Error (AMRE) and variance $\left(R^{2}\right)$ given as follows:

$$
\begin{aligned}
\mathrm{AMRE} & =\frac{1}{n} \sum_{i=1}^{n} \frac{100\left|a_{i}-y_{i}\right|}{a_{i}}, \\
R^{2} & =1-\frac{\sum_{i=1}^{n}\left(a_{i}-y_{i}\right)^{2}}{\sum_{i=1}^{n}\left(y_{i}\right)^{2}},
\end{aligned}
$$

where $a_{i}$ is the actual value, $y_{i}$ is the output value, and $n$ is the data value number.

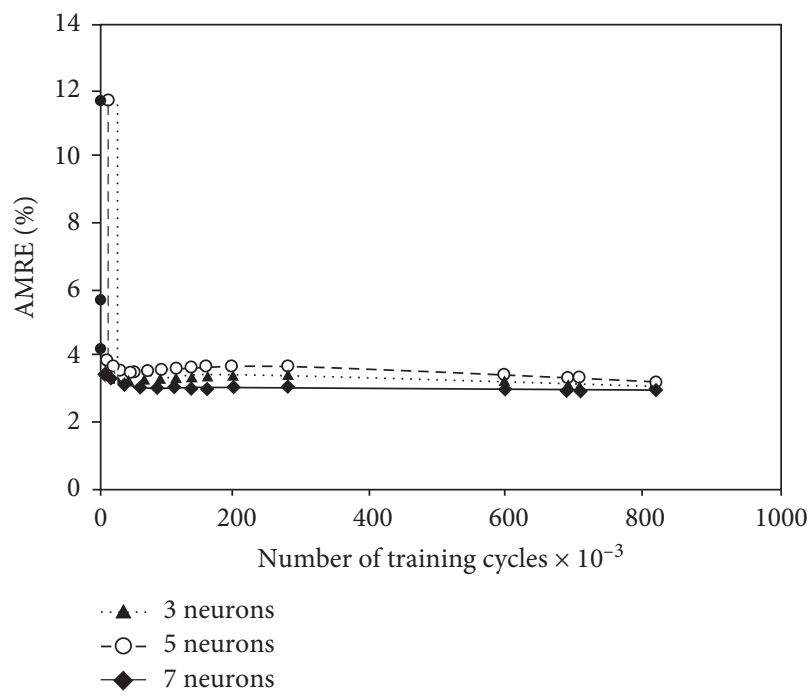

FIgURE 11: Proposed method with different neurons in hidden layers. 
TABLE 2: Absolute mean square error and absolute fraction variance for renewable energies in the DNN

\begin{tabular}{lcc}
\hline Energy sources & AMRE & $R^{2}$ \\
\hline Wind consumption & 6.58 & 0.9935 \\
Solar consumption & 5.46 & 0.9941 \\
Hydro consumption & 3.62 & 0.9991 \\
Biomass consumption & 2.84 & 0.9918 \\
\hline
\end{tabular}

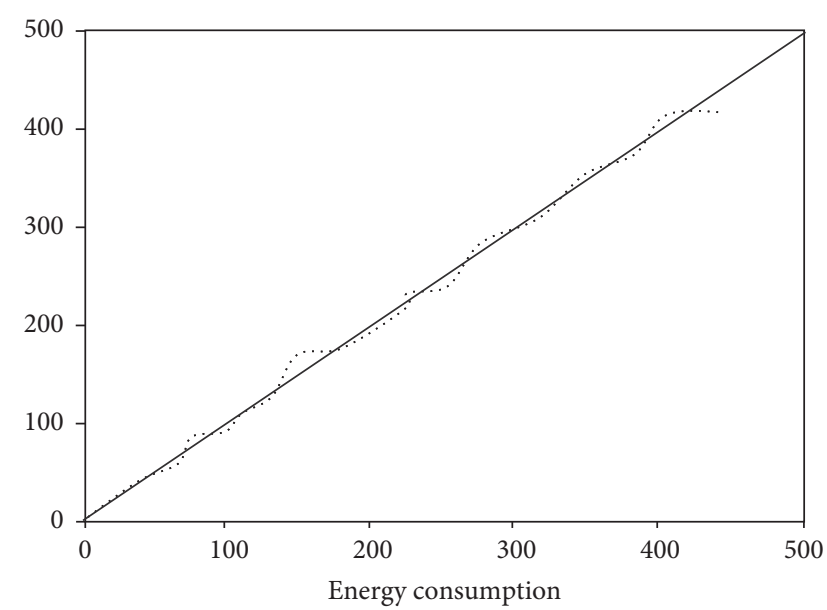

FIGURE 12: Green energy consumption within the DNN.

The result of the training system is shown in Table 1.

Evaluation of renewable green energy such as wind and solar in the DNN used three, five, and seven extra neurons with fixing a hidden layer, and the result behaves stable due to fixed features during feed forwarding and back propagation stages, as shown in Figure 11.

The DNN gets a worthy result with green energy and gains good prediction when training; four types of green energy are used in result evaluation such as hydro, wind, solar, and biomass. We calculate the AMRE and $R^{2}$ for all, as listed in Table 2.

For more details, we depict the evaluation of the deep learning system in a graph including energy consumption, as shown in Figure 12.

\section{Conclusions}

The traditional energy sources are good, but they have disadvantages. These disadvantages are that they pollute the environment through carbon emission. In addition, they are depleted, and since the increasing demand for energy is a result of the increase in the population, it is worthwhile for us to find alternatives to that energy. Green energy is one of the best alternatives that are environmentally friendly and not exhaustive. Since the issue of alternative energy is somewhat new, there must be proper management for it and associated technological development. The deep learning algorithm is considered one of the important algorithms in many applications, which has shown its worth, especially in developing green energy. In the proposed method, the deep neural network is used by extracting the features, and the deep neural network can be developed using the new features and increasing the number of hidden layers as well as feed forward and back propagation. Prediction of future consumption for green energy is the main objective in this study. Many parameters are used for the evaluation and training system based on the dataset collected from previous years. Worthy results were achieved in terms of prediction and control of energy consumption with accuracy. New parameters need to be discovered in terms of features to learn the system in the future work and use a lot of features with a classifier such as a hybrid classifier.

\section{Data Availability}

All data used to support the findings of this study are included within the article.

\section{Conflicts of Interest}

The authors confirm that there are no conflicts of interest regarding the study of this paper.

\section{Acknowledgments}

The authors are grateful to the National Natural Science Foundation of China (71971003, 71704002, and 51774013), National Social Science Fund (20ZDA084); and Humanities and Social Science Research Project of Anhui University "GA-BP-Based Deep Coal Mine Disaster Evaluation" for supporting this work.

\section{References}

[1] C. H. A. Tsang, J. Xuan, H. Wang, and D. Y. C. Leung, "Graphene materials in green energy applications: recent development and future perspective," Renewable and Sustainable Energy Reviews, vol. 120, p. 109656, 2020.

[2] F. Huang and N. Yoshino, "Sustainable solutions for green financing and investment in renewable energy projects," Energies, vol. 13, no. 4, p. 788, 2020.

[3] C. Rizakulyevna, "Alternative energy sources," The American Journal of Applied Sciences, vol. 3, pp. 58-68, 2021.

[4] S. Zangoei, N. Salehnia, and M. Khodaparast Mashhadi, "A comparative study on the effect of alternative and fossil energy consumption on economic growth and foreign direct investment in selected countries using SUR approach," Environmental Science and Pollution Research, vol. 28, no. 16, pp. 19799-19809, 2021.

[5] A. Nwozor, "Nigeria's quest for alternative clean energy development: a cobweb of opportunities, pitfalls and multiple dilemmas," Energy Policy, vol. 149, Article ID 112070, 2021.

[6] C. Bhowmik, S. Bhowmik, and A. Ray, "Green energy sources selection for sustainable planning: a case study," IEEE Transactions on Engineering Management, 2020.

[7] I. Usman, "Developing green operations to minimize energy consumption by pdca cycle of iso 50001. A case study with delphi method approach," Journal of Security \& Sustainability Issues, vol. 9, 2020.

[8] M. Skordoulis, S. Ntanos, and G. Arabatzis, "Socioeconomic evaluation of green energy investments," International Journal of Energy Sector Management, 2020.

[9] A. Rehman et al., "Asymmetric investigation to track the effect of urbanization, energy utilization, fossil fuel energy and $\mathrm{CO} 2$ 
emission on economic efficiency in China: another outlook," Environmental Science and Pollution Research, vol. 28, no. 14, pp. 17319-17330, 2021.

[10] S. Hao, A. T. H. Kuah, C. D. Rudd et al., "A circular economy approach to green energy: wind turbine, waste, and material recovery," Science of the Total Environment, vol. 702, Article ID 135054, 2020.

[11] Q. Kuah, “Analyzing the sustainability of China's industrial sectors: a data-driven approach with total energy consumption constraint," Ecological Indicators, vol. 122, Article ID 107235, 2021.

[12] R. Talat, "A decentralized system for green energy distribution in a smart grid," Journal of Energy Engineering, vol. 146, Article ID 04019036, 2020.

[13] Y. Kong, C. Feng, and J. Yang, "How does China manage its energy market? A perspective of policy evolution," Energy Policy, vol. 147, p. 111898, 2020.

[14] M. Amyotte and M. Ordonez, "Power loss prediction for distributed energy resources: rapid loss estimation equation," IEEE Transactions on Industrial Electronics, vol. 68, pp. 2289-2299, 2020.

[15] IPCC, "Potential of renewable energy outlined in report by the intergovernmental panel on climate change," 2011, http:// srren.ipcc-wg3.de/press/content/srren-press-releaseupdated-version.pdf.

[16] V. Gaigalis and V. Katinas, "Analysis of the renewable energy implementation and prediction prospects in compliance with the EU policy: a case of Lithuania," Renewable Energy, vol. 151, pp. 1016-1027, 2020.

[17] G. W. Chang and H.-J. Lu, "Integrating gray data preprocessor and deep belief network for day-ahead PV power output forecast," IEEE Transactions on Sustainable Energy, vol. 11, pp. 185-194, 2018.

[18] L. Li, Z. Yuan, and Y. Gao, "Maximization of energy absorption for a wave energy converter using the deep machine learning," Energy, vol. 165, pp. 340-349, 2018.

[19] H. Wang, H. Yi, J. Peng et al., "and probabilistic forecasting of photovoltaic power based on deep convolutional neural network," Energy Conversion and Management, vol. 153, pp. 409-422, 2017.

[20] A. LiuWang, V. Srikumar, and A. D. Smith, "Predicting electricity consumption for commercial and residential buildings using deep recurrent neural networks," Applied Energy, vol. 212, pp. 372-385, 2018.

[21] X. Luo, J. Sun, L. Wang et al., "Short-term wind speed forecasting via stacked extreme learning machine with generalized correntropy," IEEE Transactions on Industrial Informatics, vol. 14, no. 11, pp. 4963-4971, 2018.

[22] B. Sun and T. Durga, "Deep learning framework to forecast electricity demand," Applied Energy, vol. 238, pp. 1312-1326, 2019.

[23] H. Haochen, Y. Qin, H. Chuantong, and J. Cao, “Optimal energy management strategies for energy Internet via deep reinforcement learning approach," Applied Energy, vol. 239, pp. 598-609, 2019.

[24] S. Sobri and S. Koohi-Kamali, "Solar photovoltaic generation forecasting methods: a review," Energy Conversion and Management, vol. 156, pp. 459-497, 2018.

[25] B. Rahim, R. Sumedha, and A. Ghosh, "Very short-term photovoltaic power forecasting with cloud modeling: a review," Renewable and Sustainable Energy Reviews, vol. 75, pp. 242-263, 2017.
[26] C. Gallego-Castillo and A. Cuerva-Tejero, "A review on the recent history of wind power ramp forecasting," Renewable and Sustainable Energy Reviews, vol. 52, pp. 1148-1157, 2015.

[27] Y. Lopez-Garcia, P. N. Suganthan, and N. Srikanth, "Ensemble methods for wind and solar power forecasting-A stateof-the-art review," Renewable and Sustainable Energy Reviews, vol. 50, pp. 82-91, 2015.

[28] J. M. Torres, R. M. Aguilar, and K. V. Zuñiga-Meneses, "Deep learning to predict the generation of a wind farm," Journal of Renewable and Sustainable Energy, vol. 10, Article ID 013305, 2018.

[29] S. Al-Janabi, A. F. Alkaim, and Z. Adel, "An Innovative synthesis of deep learning techniques (DCapsNet \& DCOM) for generation electrical renewable energy from wind energy," Soft Computing, vol. 24, no. 14, pp. 10943-10962, 2020.

[30] H.-Z. Wang, G.-B. Wang, J.-C. Peng, H. Jiang, and Y.-T. Liu, "Deep learning based ensemble approach for probabilistic wind power forecasting," Applied Energy, vol. 188, pp. 56-70, 2017.

[31] M. Li and I. Ahmad, "Solar power generation forecasting using ensemble approach based on deep learning and statistical methods," Applied Computing and Informatics, 2020.

[32] M. Afrasiabi, M. Rastegar, and S. Afrasiabi, "Deep learning architecture for direct probability density prediction of smallscale solar generation," IET Generation, Transmission \& Distribution, vol. 14, no. 11, pp. 2017-2025, 2020.

[33] D. Prado and M. Lopez, Advances in Financial Machine Learning, John Wiley and Sons, New York, NY, USA, 2018.

[34] G. Sulong and A. Mohammedali, "Human activities recognition via features extraction from skeleton," Journal of Theoretical \& Applied Information Technology, vol. 68, p. 3, 2014.

[35] E. Hassan, Y. Khalil, and I. Ahmad, "Learning feature fusion in deep learning-based object detector," Journal of Engineering, vol. 2020, 2020.

[36] G. Sulong and A. Mohammedali, "Recognition of human activities from still image using novel classifier," Journal of Theoretical \& Applied Information Technology, vol. 71, p. 1, 2015.

[37] E. Hassan, Y. Khalil, and I. Ahmad, "Learning feature fusion in deep learning-based object detector," Journal of Engineering, vol. 202011 pages, Article ID 7286187, 2020.

[38] B. T. Atiyha, S. Aljabbar, and A. Jaber, "An improved cost estimation for unit commitment using back propagation algorithm," Malaysian Journal of Fundamental and Applied Sciences, vol. 15, no. 2, pp. 243-248, 2019.

[39] D. Ali, L. Zhang, and Li Bai, "Deep learning in visual computing and signal processing," Applied Computational Intelligence and Soft Computing, p. 2017, 2017.

[40] S. O. Geoffrey, E. Hinton, and Y.-W. Teh, "A fast learning algorithm for deep belief nets," Neural Computing and Applications, vol. 18, no. 7, pp. 1527-1554, 2006.

[41] K. Wang, X. Qi, H. Liu, and J. Song, "Deep belief network based k-means cluster approach for short-term wind power forecasting," Energy, vol. 165, pp. 840-852, 2018.

[42] C. Yu, Y. Li, Y. Bao, H. Tang, and G. Zhai, "A novel framework for wind speed prediction based on recurrent neural networks and support vector machine," Energy Conversion and Management, vol. 178, pp. 137-145, 2018. 